\title{
Terapia ocupacional em contextos hospitalares: a especialidade, atribuições, competências e fundamentos
}

\author{
Occupational therapy in hospital contexts: the specialty, duties, skills and \\ fundamentals
}
Terapia ocupacional en contextos hospitalarios: la especialidad, atribuciones, competencias y fundamentos

\section{Recebido: 10/04/2017 \\ Aprovado: 15/08/2017 \\ Publicado: 30/03/2018}

\author{
Heloísa Cristina Figueiredo Frizzo ${ }^{1}$ \\ Victor Augusto Cavaleiro Corrêa ${ }^{2}$
}

Trata-se de uma reflexão que teve como objetivo contextualizar a atuação da Terapia Ocupacional em contextos hospitalares. Muitos são os referenciais teóricos observados na prática terapêutica ocupacional neste campo, dentre eles destacam-se a estrutura da prática da Terapia Ocupacional: domínio e processo, e a Ciência Ocupacional ou Ciência da Ocupação. Independentemente dos referenciais teóricos que subsidiam a atuação do profissional especialista em contextos hospitalares, espera-se que as habilidades e competências do terapeuta ocupacional não se restrinjam a visão fragmentada do conhecimento em subespecialidades clássicas da formação, tais como: saúde mental e reabilitação física, uma vez que o cuidado à pessoa em processo de adoecimento e hospitalização, exige uma visão integral e ampliada da pessoa e sua relação com as ocupações num cotidiano singular.

Descritores: Terapia ocupacional; Assistência hospitalar; Cuidados paliativos.

It is a reflection that aimed to contextualize the work of occupational therapy in hospital contexts. There are many theoretical references in occupational therapy practice in this field, including the occupational therapy practice framework: domain and process, and Occupational Science or Science of the Occupation. Regardless of the theoretical references that subsidize the professional specialist in hospital contexts, the skills and competencies of the occupational therapist is not restricted to a fragmented vision of knowledge in classic training subspecialties, such as: mental health and physical rehabilitation, since the person care in the process of illness and hospitalization, requires an integral and expanded vision of the individual and their relationship with the occupations in everyday life.

Descriptors: Occupational therapy; Hospital care; Palliative care.

Se trata de una reflexión con el objetivo de contextualizar la actuación de la Terapia Ocupacional en contextos hospitalarios. Muchos son los referenciales teóricos observados en la práctica terapéutica ocupacional en este campo, dentro de los que se destacan la estructura de la práctica de la Terapia Ocupacional: dominio y proceso, y la Ciencia Ocupacional o Ciencia de la Ocupación. Independientemente de los referenciales teóricos que subsidian la actuación del profesional especialista en contextos hospitalarios, se espera que las habilidades y competencias del terapeuta ocupacional no se restrinjan a una visión fragmentada del conocimiento en subespecialidades clásicas de la formación, como: salud mental y rehabilitación física, ya que el cuidado a la persona en proceso de enfermedad e intención, exige una visión integral y ampliada de la persona y su relación con las ocupaciones en un cotidiano singular.

Descriptores: Terapia ocupacional; Atención hospitalaria; Cuidados paliativos.

1. Terapeuta Ocupacional. Especialista em Administração Hospitalar. Especialista em Acupuntura. Especialista em Informação em Saúde. Mestre em Ciências Médicas em Saúde Mental. Doutora em Ciências. Pós Doutorado em Ciências, Tecnologia e Sociedade. Professora Adjunta do curso de Terapia Ocupacional da Universidade Federal do Triângulo Mineiro, Uberaba, MG, Brasil. ORCID: 0000-0002-7661-0353 E-mail: heloisa.frizzo@yahoo.com.br

2. Terapeuta Ocupacional. Especialista em Saúde da Família. Mestre em Psicologia. Doutor em Doenças Tropicais. Professor Adjunto da Faculdade de Fisioterapia e Terapia Ocupacional da Universidade Federal do Pará, Belém, PA, Brasil. ORCID: 0000-0003-0133-7927

E-mail: victorcavaleiro@gmail.com 


\section{INTRODUÇÃO}

A criação das Especialidades em Terapia Ocupacional é um fato relativamente recente datando do final da última década, mais precisamente em 20091.

Este cenário é visto atualmente por uma parcela de profissionais como uma resultante da mobilização da categoria frente às necessidades e realidades do mercado de trabalho, e delimitando consequentemente campos e núcleos de competências de atuação profissional.

Este movimento tem sido legitimado pelo Conselho Federal de Fisioterapia e Terapia Ocupacional (COFFITO) em parceria com os Conselhos Regionais e Associações e entidades representativas de classe, a saber Associação Brasileira de Terapeutas Ocupacionais e (ABRATO) e Associação Científica em Contextos Hospitalares e Cuidados Paliativos (ATOHOSP). Em contrapartida, a definição de especialidades em Terapia Ocupacional é questionada por muitos profissionais terapeutas ocupacionais em função do raciocínio disciplinar, fragmentado e reducionista.

A busca da legitimação dos campos e núcleos de competência, e da consolidação do conhecimento específico/especializado das especialidades nem sempre perpassa o diálogo e a reflexão com as políticas públicas na área de saúde, educação, social e cultura, na busca de consonância com as diretrizes curriculares nacionais para os cursos de graduação que preconiza a formação do profissional generalista, humanista e com os fundamentos e princípios da profissão. Essa realidade, se não observada com critério, pode implicar na fragmentação do conhecimento e das práticas em Terapia Ocupacional.

A constituição das especialidades se organiza segundo um jogo de negociações provisórias, em certa medida intermináveis porque são impossíveis de serem arbitradas exclusivamente por uma racionalidade técnica ${ }^{2}$. A definição destes campos depende também de interesses políticos, profissionais mais amplos e não apenas de diretrizes de cada categoria profissional ${ }^{2}$.

$\begin{array}{cl}\text { Em relação à área de atuação da } \\ \text { Terapia } & \text { Ocupacional em contextos }\end{array}$ hospitalares, nota-se reconhecimento da Especialidade em 06 de novembro de 2009, a partir da Resolução COFFITO no 371 - 06 de novembro de 20091, que dispõe sobre a alteração do artigo 1을 da Resolução COFFITO no 366/2009, que não contemplava a referida especialidade. Este documento reconhece assim, as especialidades em Terapia Ocupacional: Saúde Mental; Saúde Funcional; Saúde Coletiva; Saúde da Família; Contextos Sociais; Contextos Hospitalares e Acupuntura. No entanto, a disciplinarização da especialidade ocorreu somente em julho de 2013, a partir da Resolução no. 429, que define as áreas de atuação e competências do terapeuta ocupacional especialista em Contextos Hospitalares ${ }^{3}$.

Este estudo teve como objetivo contextualizar a atuação da Terapia Ocupacional em contextos hospitalares.

\section{MÉTODO}

Trata-se de um reflexão acerca da atuação da Terapia Ocupacional em Contextos Hospitalares, com foco na regulamentação da especialidade no Brasil, as áreas de atuação do profissional, e respectivas atribuições e competências, assim como fundamentos para a prática.

Utilizou-se análise crítica e reflexiva das Resoluções do Conselho Federal de Fisioterapia e Terapia Ocupacional (COFFITO), documentos estes disponíveis online em acesso aberto (https://www.coffito.gov.br/nsite/) e da literatura especializada na área de atuação da Terapia Ocupacional em assistência hospitalar. Os dados disponíveis foram analisados e fundamentados a partir de revisão bibliográfica.

Como base se usou os seguintes questionamentos: Como se dá a inserção do terapeuta ocupacional na assistência hospitalar atualmente no Brasil? 0 que preconiza a especialidade Terapia Ocupacional em Contextos Hospitalares e Cuidados Paliativos conforme preconizado pelo COFFITO? Quais são as áreas consideradas de atuação do terapeuta ocupacional especialista em Contextos Hospitalares? Quais são as atribuições e competências desse profissional nessa 
especialidade? Quais fundamentos subsidiam a sua prática em Contextos Hospitalares e Cuidados Paliativos?

\section{RESULTADOS}

A partir dos questionamentos supracitados e com base na revisão de bibliografias afins acerca da regulamentação da Especialidade Terapia Ocupacional em Contextos Hospitalares e Cuidados Paliativos, criaram-se três eixos temáticos, a saber:

1) "A regulamentação, atribuições, competências do terapeuta ocupacional especialista em Contextos Hospitalares e Cuidados Paliativos";

2) "Fundamentos para a prática em Terapia Ocupacional em Contextos Hospitalares e Cuidados Paliativos"; e,

3) "Reflexões acerca da atuação da Terapia Ocupacional em Contextos Hospitalares e Cuidados Paliativos".

\section{DISCUSSÃO}

A regulamentação, atribuições, competências do terapeuta ocupacional especialista em Contextos Hospitalares e Cuidados Paliativos.

A disciplinarização da especialidade Contextos Hospitalares ocorreu em julho de 2013, a partir da Resolução $n^{\circ}$. 429, que define as áreas de atuação e competências do terapeuta ocupacional especialista em Contextos Hospitalares ${ }^{3}$.

Para o COFFITO ${ }^{1}$, são consideradas áreas de atuação do especialista em Contextos Hospitalares: Intra-Hospitalar, ExtraHospitalar e Cuidados Paliativos. Nesse sentido, são considerados ambientes de atuação da especialidade: os hospitais, os ambulatórios (unidades especializadas, clínicas, consultórios e centros de saúde), os domicilios e os serviços de home care, podendo estes serem de caráter público, privado, filantrópico, militar ou terceiro setor.

As intervenções nessa especialidade preconiza: o diagnóstico terapêutico ocupacional, a eleição, execução e utilização de métodos, técnicas e recursos pertinentes e adequados aos contextos hospitalares, intervindo junto ao indivíduo e coletividade (cliente/paciente/usuário, cuidadores e grupos), em todos os níveis de atenção e fases do desenvolvimento ontogênico ${ }^{1}$.

Assim, a referida resolução prevê que o terapeuta ocupacional especialista em Contextos Hospitalares deverá promover ações de promoção, prevenção, proteção, educação, intervenção, recuperação, reabilitação e cuidados paliativos, segundo princípios e diretrizes do Sistema Único de Saúde, em especial em relação às concepções da integralidade e humanização da atenção à saúde, com destaque para o cuidado oferecido em linhas de cuidado ${ }^{1}$.

As atribuições exercidas pelo Especialista terapeuta ocupacional em Contextos Hospitalares abrangem o gerenciamento de serviços, o ensino e pesquisa, cabendo ao profissional à responsabilidade técnica, coordenação e supervisão, gestão, direção, chefia, consultoria, auditoria, perícia, ensino e pesquisa ${ }^{1}$.

Nesse sentido, espera-se que o terapeuta ocupacional especialista em Contextos Hospitalares seja capaz de: realizar consulta, interconsulta e avaliação terapêutica ocupacional com paciente, cliente, usuário, família, cuidadores e grupos; estabelecer diagnóstico Terapêutico Ocupacional e se necessário solicitar interconsulta, exames complementares e pareceres para definir a conduta e o prognóstico terapêutico-ocupacional; realizar o planejamento do tratamento e intervenção constituída por uma série de ações que envolvem tanto a seleção, como a indicação e aplicação de métodos, técnicas e procedimentos terapêuticos ocupacionais, adequados e pertinentes às necessidades e características do paciente/cliente/usuário dos familiares, cuidadores e grupos, monitorando seu desempenho nas diferentes áreas ocupacionais ${ }^{1}$.

Particularmente se foca nas Atividades de Vida Diária (AVD's), Atividades Instrumentais de Vida Diária (AIVD's), produtividade, lazer e participação social; determinar as condições de alta terapêutica ocupacional e possíveis encaminhamentos; emitir laudos, atestados, pareceres e relatórios terapêuticos ocupacionais; 
participar de órgãos gestores, gerenciar áreas técnicas e administrativas ${ }^{1}$.

\section{Fundamentos para a prática em Terapia Ocupacional em Contextos Hospitalares e Cuidados Paliativos}

Muitos referenciais têm sido observados na prática terapêutica ocupacional neste campo.

Em relação a esta questão, as ações da Terapia Ocupacional devem garantir ao sujeito e a sua rede social de serem compreendidos e cuidados a partir de uma escuta mais ampla de suas necessidades, evitando-se assim tanto abordagens teóricometodológicas adotadas a priori (a partir da opção de especialidade do terapeuta ocupacional), como racionalidades mais idealizadas do sistema de saúde e menos próximas das necessidades dos seus usuários, considerando-se inclusive o hospital como um lugar para a promoção à saúde 4 .

Diversos referênciais teóricos têm sido utilizados para nortear a prática terapêutica ocupacional junto aos contextos hospitalares e cuidados paliativos. Dentre elas, a estrutura da prática da Terapia Ocupacional: domínio e processo, proposto pela Associação Americana de Terapia Ocupacional (AOTA), $3^{\text {a }}$. Edição ${ }^{5}$, podendo esta abordagem nortear os princípios e diretrizes da Resolução no. 429, de 2013, que define a atuação do terapeuta ocupacional especialista em Contextos Hospitalares.

A AOTA ${ }^{5}$ compreende que o domínio da Terapia Ocupacional visa apoiar o envolvimento, a participação e a saúde. São focos de atenção do domínio da Terapia Ocupacional as ocupações, os fatores do cliente, as habilidades do desempenho, os padrões do desempenho além dos contextos e ambientes, enquanto o processo terapêutico é ordenado por meio da avaliação, intervenção e monitoramento dos resultados.

Outro referencial teórico possível para subsidiar as práticas na Terapia Ocupacional em Contextos Hospitalares é a Ciência Ocupacional ou Ciência da Ocupação que compreende os fenômenos numa concepção multicausal e relacional, considerando as interações como dinâmicas, sendo consideradas pelo prisma da complexidade ${ }^{6}$, em que ocupar-se é compreendido como uma ação que assume uma forma, um sentido e um significado para uma determinada pessoa, que se encontra em um determinado contexto físico, social, cultural, econômico.

Esta é uma configuração de cunho individual e subjetivo, complexo e que é influenciada por diversos fatores, que interagem de forma não-linear e dinâmica.

Sabe-se que a forma ocupacional compreende o que as pessoas fazem e como ocorre em relação ao tempo e ao espaço. Resulta da interação de uma atividade com o ambiente em determinado tempo e espaço físico e social. A forma faz referência às características visíveis e observáveis de uma ocupação $0^{6,7}$.

Busca-se compreender o que e como as pessoas fazem? Como preenchem o tempo? No contexto hospitalar e nos cuidados paliativos, a forma como se apresentam as ocupações rotineiras podem estar modificadas.

Ainda no que diz respeito à ocupação, a Sociedade Internacional de Cientistas Ocupacionais ${ }^{8}$, acredita no valor da ocupação e afirma que "a ocupação engloba todas as atividades humanas, quer sejam físicas, mentais, sociais e espirituais, sendo fundamental para autonomia, saúde, bemestar e da justiça (p.2)".

Nesse sentido, as ocupações são os fazeres diários dos seres humanos, as quais possuem uma maneira típica de ocorrer na vida de cada pessoa, agregando e ancorando significados íntimos e específicos de cada um que executa um determinado fazer, os quais, por sua vez, são reconhecidos e organizados pelas pessoas em contextos temporal, cultural e social ${ }^{\text {. }}$.

As ocupações também podem ser compreendidas como algo que pode gerar impactos negativos para a pessoa ${ }^{9}$. Dessa forma, como deve ser se ocupar ou dar prosseguimento as ocupações diárias, diante de um adoecimento ou frente a uma ondição crônica ou evolutiva de enfermidade?

Existe uma necessidade de conhecer a ocupação conforme a experiência de quem a realiza. Esta indicação impulsiona a pensar e a necessidade de compreender como as 
ocupações das pessoas variam ou se apresentam em relação a cada tempo, cultura, meio social e momento de vida de cada um, a partir de uma compreensão da forma, da função e do significado ocupacional ${ }^{10}$.

Assim, se faz necessário examinar o que os seres humanos fazem com o seu tempo, compreender sua variação de acordo com a habilidade de cada pessoa, como organizam as atividades e suas adaptações ao meio, seus objetivos, o que significam, qual o valor, identificar como as ocupações funcionam em um determinado momento de vida de uma pessoa, entre outras. Esses questionamentos conduzem a compreensão de uma dimensão ocupacional.

A centralidade do engajamento das pessoas em ocupações e na vida humana, principalmente como elas se relacionam com a saúde, o bem estar e a participação social ${ }^{11}$.

Neste sentido, o foco não deve estar somente nas atividades em si, mas no significado que são atribuídos a elas. Observase o individuo inserido em seu contexto, em torno de suas ocupações, analisando as atividades e as relações que se estabelecem no processo de criação, adaptação, construção e reconstrução, ou seja, um ser que se ocupa em interação com os contextos.

\section{Reflexões acerca da atuação da Terapia Ocupacional em Contextos Hospitalares e Cuidados Paliativos}

Independentemente dos referenciais teóricos que subsidiam a atuação do profissional especialista em Contextos Hospitalares e Cuidados Paliativos, espera-se que suas habilidades e competência não se restrinjam a uma visão fragmentada do conhecimento em subespecialidades clássicas da formação em Terapia Ocupacional, tais como saúde mental e reabilitação física.

Espera-se o cuidado à pessoa em processo de adoecimento, hospitalização e eminência de morte e suas repercussões biopsicossociais, para além de um modelo centrado na doença e resgate das habilidades e funções comprometidas/perdidas.

As ações a serem desenvolvidas buscam uma abordagem ampliada da clínica, que não está focada na doença, nos sinais e sintomas e sim na compreensão da pessoa em sua singularidade, na proposição de projetos terapêuticos individuais, que visam à autonomia, a tomada de decisão e a participação, no desenvolvimento de ações de cuidado integral e humanizado.

Além disso, deve ser capaz de considerar as dimensões da macro instituição hospital em relação ao processo de cuidado, que impõe uma lógica positivista, normativa e pouco singular, compreendendo o hospital como uma unidade de cuidado, inserido em uma perspectiva mais ampla que incluí a comunidade e o ambiente no qual a pessoa está inserida.

A perspectiva do processo de trabalho centrado na clínica ampliada possibilita a ampliação do grau de autonomia e da capacidade dos usuários compreenderem e atuarem sobre si mesmo e sobre o mundo da vida, entendendo-se que o grau de autonomia se mede pela capacidade de autocuidado, de compreensão sobre o processo saúde/enfermidade, pela capacidade de usar o poder e de estabelecer compromisso e contrato com outros ${ }^{12}$.

A alteração do "objeto" e do "objetivo" do trabalho clínico exigirá mudança nos meios de intervenção, sejam eles diagnósticos ou terapêuticos. A terapêutica não se restringe somente a fármacos e à cirurgia, mas aos recursos terapêuticos que valorizam a escuta e da palavra, o poder da educação em saúde e o apoio psicossocial ${ }^{12}$.

Nesse processo para alcançar a capilaridade é importante que cada hospital incorpore em seu cotidiano dispositivo de educação continuada, que possibilite uma reconstrução de conhecimentos e das posturas da maioria de seus trabalhadores ${ }^{13}$.

Aponta também que um desses recursos para esta perspectiva de cuidado é a elaboração de Projetos Terapêuticos Singulares. Esses projetos têm por objetivo a realização de uma revisão do diagnóstico, nova avaliação de riscos e uma redefinição das linhas de intervenção terapêutica, redefinindo tarefas e encargos dos vários especialistas ${ }^{14}$.

A atuação do terapeuta ocupacional junto ao hospital deve buscar desenvolver escuta e acolhimento às necessidades e 
problemáticas dos sujeitos sob cuidado, deslocando o hospital do eixo da assistência à saúde e entendendo-o como estação do cuidado 4 .

Ao oferecer atenção a partir de linhas do cuidado (à criança e ao adolescente; ao adulto sob cuidados clínicos e cirúrgicos; à saúde materno-infantil; à pessoa com HIV/AIDS, à pessoa com câncer; à pessoa em cuidados paliativos, entre outras), as práticas e saberes produzidos pela Terapia Ocupacional poderão percorrer itinerários nas diferentes linhas passando pela atenção primária, secundária e terciária, e parando em várias 'estações do cuidado'4.

Nesta perspectiva, o foco das ações do terapeuta ocupacional no hospital sejam as atividades e cotidianos. 0 cuidado à saúde que a Terapia Ocupacional disponibiliza se constitui em poder entender e intervir nas manifestações e descontinuidades da cotidianidade ocasionadas por situações diversas de adoecimento, que transitam pelo domicílio, hospital, e/ou por outros equipamentos sociais e de saúde ${ }^{4}$.

Esta visão ampliada e generalista dos processos de adoecimento e hospitalização, na perspectiva ampliada e na desconstrução do modelo institucional biomédico, não nos isenta do domínio, de habilidades e competências técnicas relacionadas às demandas particulares de clínicas complexas e específicas, que demandam olhares peculiares.

Unidades de terapia intensiva, centros de terapia renal, unidades de tratamento oncológico, unidades de pediatria e outras mais demandam conhecimentos e habilidades afins. Esta realidade nos alerta para o fato de que oferecer interconsulta não garante uma abordagem específica e particularizada. Para este fim faz-se necessário a participação mais ativa e continua do profissional terapeuta ocupacional junto a equipe e unidade de referencia da área solicitada.

A interconsulta tem como objetivos auxiliar profissionais de outras áreas no diagnóstico e tratamento de pacientes com problemas psicossociais (situações emocionais emergentes) e intermediar a relação entre os envolvidos na situação (equipe de saúde, pacientes e familiares), facilitando a comunicação, a cooperação e a elaboração de conflitos ${ }^{15}$.

No Brasil, um primeiro estudo realizado na área foi produzido junto a um serviço de interconsulta psiquiátrica de um hospital de clínicas do interior do estado de São Paulo. 0 presente estudo teve como objetivo identificar os motivos de solicitação de interconsulta direcionados pela equipe de interconsulta psiquiátrica para o atendimento em Terapia Ocupacional. Evidenciou-se que os principais motivos de solicitação estavam relacionados às dificuldades atribuídas pela equipe em relação ao paciente, seguidos de dificuldades relacionadas ao apoio à equipe ${ }^{16}$.

Dentre os motivos atribuídos aos pacientes destacam-se: as manifestações de sintomatologias psiquiátricas, a solicitação de seguimento psicossocial/psicoterápico; as reações emocionais decorrentes de procedimentos invasivos; os antecedentes psiquiátricos, e disfunções, incapacidades ou deficiência física. As solicitações de seguimento psicossocial/psicoterápico referiam-se principalmente ao apoio a pessoas com prognóstico ruim, em terminalidade de vida ${ }^{16}$.

Quanto aos motivos relacionados à equipe, foram observadas as dificuldades de manejo, solicitação de apoio ao diagnóstico diferencial e investigação diagnóstica. A resistência ao tratamento e o isolamento/restrição ao leito também foram motivos para a solicitação de interconsulta, além do hospitalismo e dilemas éticos. Os principais dilemas éticos identificados foram: abuso sexual extra-hospitalar, desejos de eutanásia, abandono familiar e apoio na comunicação de diagnósticos e notícias difíceis. No momento de realização desta pesquisa, poucas eram as práticas de Terapia Ocupacional em Hospitais Gerais com registros e produções científicas afins ${ }^{16}$.

Outro estudo ${ }^{17}$ aponta que os motivos para solicitação de inteconsulta em Terapia Ocupacional em Hospital Geral estão relacionados a períodos longos de internação; facilitação no manejo clínico com o paciente; ampliação do campo relacional (pacientefamiliar, paciente-equipe, paciente-paciente); 
e criação de condições de melhoria e adaptação do paciente aos procedimentos necessários ao tratamento e à internação.

Outra pesquisas destacam cinco grupos de motivos de solicitação de interconsulta em Terapia Ocupacional. São eles: "hospitalização", que engloba questões relacionadas à dificuldade do paciente de se adaptar à rotina hospitalar, de ficar internado por um longo período e de se relacionar com a equipe; "ociosidade", que consiste na constatação de ociosidade do paciente e na necessidade de ocupação; "outros", incluindo auxílio para as práticas de Atividades de Vida Diária (AVD's), confecções de órteses, promoção de estimulação neuropsicomotora e/ ou solicitação de Terapia Ocupacional pelos pacientes, uma vez que estes já haviam sido atendidos em internações anteriores; "aspecto emocional do paciente", ou seja, solicitação baseada em queixas ou diagnósticos psiquiátricos (como tristeza, desânimo, depressão, ansiedade); e "ausência de descrição dos motivos de solicitação"18,19.

Como funções do Terapeuta Ocupacional interconsultor em hospital geral destaca-se: ampliar os aspectos saudáveis; diminuir ruptura e desorganização da vida cotidiana; auxiliar no processo de desospitalização e reinserção do indivíduo; discutir estratégias com a equipe solicitante; e orientar $\mathrm{o}$ familiar no cuidado com o paciente 20 .

No hospital geral existe um sujeito temporariamente sem cotidiano, que pode ou não estar inserido em uma rotina hospitalar ${ }^{18,19}$.

Para evidenciar esta reflexão, há diferença entre rotina no contexto hospitalar e cotidiano. A rotina é um caminho já conhecido, uma sequência de atos, usos, observada por força do hábito, enquanto o cotidiano é a vida de todo homem, sem nenhuma exceção, qualquer que seja seu posto na divisão do trabalho intelectual e físico; e a vida cotidiana, é heterogênea em grande parte e sob vários aspectos, sobretudo no que se refere ao conteúdo e à significação de nossos tipos de atividades ${ }^{18,21}$.

A rotina é uma sequência de atos desprovidos de significados e que o cotidiano, por outro lado, constitui-se por essa particularidade dos significados atribuídos às ações pelo sujeito ${ }^{18}$.

Assim, como o cotidiano está relacionado com a participação social, pode-se pensar que no ambiente hospitalar, os terapeutas ocupacionais vão lidar diretamente com a rotina e não com o cotidiano do indivíduo, porém podem fornecer um espaço de vivência de novas capacidades que vão auxiliar o indivíduo a reconstruir, retomar ou criar seu cotidiano, já que "(a organização de cotidianos) tem início desde que uma relação se instale na transferência, permitindo que, através de atividades construídas, esse cotidiano seja significado para o sujeito em Terapia Ocupacional".

Destaca-se aqui outra perspectiva de se pensar o cotidiano nos contextos hospitalares a partir da definição de "cotidiano", como "cada dia", como a unidade de medida da sucessão da vida humana feita de um dia após o outro de forma fluida, sucessiva e continuada; acrescentando que o cotidiano inclui o indivíduo no plano da vida em comum com os outros, que integra o indivíduo na comunidade ${ }^{22}$.

Assim, adoecer e estar hospitalizado tem relação direta no viver de cada dia na vida humana, tendo em vista que esta experiência restringe uma vida de forma fluida, sucessiva e continuada, limitando e ameaçando o viver, em um dos momentos mais vulneráveis na pessoa. Faz-se necessário a reconstrução deste cotidiano ainda que em condições adversas, onde perpetua a rotina tão imposta, porém necessária à continuidade da vida.

Já em uma perspectiva ancorada na Ciência Ocupacional, a ocupação circunscreve a Terapia Ocupacional, em que a atuação profissional vai fundamentar suas intervenções sob o fazer humano e suas repercussões na vida, conduzindo-se para uma forma de dimensionar, perceber, interpretar e intervir com o seu fazer. Nesta perspectiva, a Terapia Ocupacional considera a ocupação como produto e meio de construção do próprio homem ${ }^{23}$.

Busca entender as relações que a pessoa estabelece com sua condição de vida e 
as ocupações, considerando estas, como instrumentos de domínio científico para compreender a ocupação humana, uma vez que as ocupações possibilitam à pessoa ser reconhecida e se reconhecer por seus afazeres, além de permitirem conhecer a história de vida e ocupacional desta e a maneira como ela as desempenha no contexto em que está inserida ${ }^{23}$.

Dependendo de como as pessoas interagem e percebem suas ocupações diárias, poderão influenciar na percepção de uma determinada identidade ocupacional e pessoal de si. Essas ocupações refletem valores culturais, fornecem estruturas, o(s) sentido(s) (os 'por quês de uma ocupação') do(s) fazer(es) que satisfaz(em) ou não as necessidades humanas ${ }^{11}$.

$\mathrm{Na}$ condição de adoecimento e hospitalização se pode exigir mudança(s) e adaptação(ões) na maneira como se relaciona, interage com o seu entorno por meio de suas ocupações ou com a necessidade de redescobrir a função e o significado de sentirse capaz.

Pode-se conduzir a uma necessidade de rever, a partir das novas condições, as habilidades para execução daquilo que lhe dá prazer e sentido a vida.

As ocupações antes, durante e depois de uma enfermidade e internação, podem encontrar-se desconectadas. Funções e ocupações diárias podem ser bruscamente alteradas, podendo ocorrer tristezas, angústias, sentimentos de inutilidade e apatia, onde a forma, o sentido e o significado do fazer e da vida podem estar modificados.

Estudos têm abordado para além das experiências individuais. Estes têm enfocado os fatores que influenciam e determinam a participação das pessoas em ocupações, destacando os aspectos positivos e negativos associados ao envolvimento e/ou privação do engajamento nessas ações. Além disso, também priorizam investigações que desvelam o compartilhamento dessas experiências e suas repercussões na coletividade ${ }^{24-27}$.

$\mathrm{Na}$ assistência e no cuidado as pessoas no contexto hospitalar e cuidados paliativos, a Terapia Ocupacional busca compreender como as pessoas se ocupam no entorno em que vivem, ou como os modos de se ocupar têm influenciado ou implicado na condição de saúde, de vida e na participação no entorno social.

Por que ou o que levam as pessoas a se ocuparem daquilo que fazem todos os dias? Será que as pessoas sempre se ocupam daquilo que querem e no momento em que desejam? E o que as pessoas pensam sobre suas ocupações diárias? São importantes? Por quê? Que significados são atribuídos?

As respostas às estas perguntas podem ajudar a entender a dimensão ocupacional das pessoas que se encontram com as vidas e as ocupações modificadas em decorrência do adoecimento e da hospitalização.

Nestas condições, é necessário estar aberto a compreender o que sustenta e/ou alimenta ou não o movimento e a intenção de se ocupar todos os dias. Essa pode semear produções de conhecimento que revelam a importância das ocupações em diferentes contextos de vidas, condição que tem influenciado a prática da Terapia Ocupacional.

\section{CONCLUSÃO}

0 terapeuta ocupacional na especialidade em Contextos Hospitalares e Cuidados Paliativos busca em conjunto com a pessoa em adoecimento e hospitalização identificar fatores facilitadores e limitadores das ocupações quando neste momento e contexto de vida.

Assim é importante à prática profissional a construção de projetos Terapêuticos Singulares juntamente com a equipe multiprofissional.

No hospital, é necessário compreender, dentre outros aspectos, de que maneira a ocupações ocorrem? Quais os sentidos e significados destas? Quais os fazeres que deixaram de ser realizados? 0 que se tornou rotineiro ou habitual no hospital, e especialmente, como a pessoa se sente e o que percebe sobre as ocupações vividas no hospital?

\begin{tabular}{lrrrr}
\multicolumn{1}{c}{ Com } & base & nisto, o & terapeuta \\
ocupacional & pode & facilitar & o & (re) \\
conhecimento do & hospital como & lugar- \\
território, de & pertencimento, & de
\end{tabular}


possibilidades, e que a mera repetição de ações diárias é ilusória, e de que o cotidiano apresenta um potencial transformador. 0 processo terapêutico ocupacional pode ser, assim, um catalisador de uma tomada de consciência que desperte para um viver mais pleno, saudável, criativo e com produção de vida.

A prática do terapeuta ocupacional pode estar voltada para as possibilidades e recursos, onde há um compromisso com a potencialização, intensificação da vida, que permite trocas e relações humanas.

Visa compreender de que forma as ocupações humanas podem ser utilizadas para reunir e (re) significar fragmentos de suas experiências e transformá-los em novos elementos, que possibilitem ampliar a vida prática e concreta, e enfrentar de modo mais ativo e saudável o período de hospitalização e/ou para além desse.

\section{REFERÊNCIAS}

1. Conselho Federal de Fisioterapia e Terapia Ocupacional. Resolução no 371, de 06 de novembro 2009. Dispõe sobre a alteração do artigo 1ำ da Resolução COFFITO no 366 [Internet]. D.O.U., Brasília, DF, 30 nov 2009 [citado em 13 ago 2017]; 228(Seção 1):852. Disponível

em: https://www.coffito.gov.br/nsite/?p=3134\#m ore-3134

2. Campos GWS, Chakour M, Santos RC. Análise crítica sobre especialidades médicas e estratégias para integrá-las ao Sistema Único de Saúde (SUS). Cad Saúde Pública. 1997; 13(1):141-4.

3. Conselho Federal de Fisioterapia e Terapia Ocupacional. Resolução no 429, de 08 de julho de 2013. Reconhece e disciplina a especialidade de Terapia Ocupacional em Contextos Hospitalares, define as áreas de atuação e as competências do terapeuta ocupacional especialista em Contextos Hospitalares e dá outras providências [Internet]. D.O.U., Brasília, DF, 02 set 2013 [citado em 13 ago 2017]; 169(Seção I). Disponível em: https://www.coffito.gov.br/nsite/?p=3191\#m ore-3191

4. Galheigo SM. Terapia ocupacional, a produção do cuidado em saúde e o lugar do hospital: reflexões sobre a constituição de um campo de saber e prática. Rev Ter Ocup. 2008; 19(1):20-8.
5. American Occupational Therapy Association. Occupational therapy practice framework: domain and process. 3rd ed. Am J Occup Ther. 2014; 68(1):1-48.

6. Carrasco J, Olivares D. Haciendo camino al andar: construcción y comprensión de la ocupación para la investigación y práctica de la terapia ocupacional. Rev Chil Ter Ocup. Dec 2008; (8):5-16.

7. Jáuregui JG, Lucero DC. Forma, función y significado de la conducción de automóviles en un grupo de adultos mayores residentes en Santiago de Chile. Rev Chil Ter Ocup. 2013; 13(1):23-32.

8. International Society of Occupational Science Disponível em: http://www.isoccsci.org. Acesso em: 13 ago. 2017.

9. Magalhães L. Ocupação e atividade: tendências e tensões conceituais na literatura anglófona da terapia ocupacional e da ciência ocupacional. Cad Ter Ocup UFSCar. 2013; 21(2):255-63.

10. Dickie V. 0 que é ocupação? In: Crepeau EB, Cohn ES, Schell BAB., editores. Willard e Spackman: Terapia ocupacional. 11 ed. Rio de Janeiro: Guanabara Koogan, 2011. p. 15- 21.

11. Clark F, Lawor MC. A elaboração e o significado da ciência ocupacional. In: Crepeau EB, Cohn ES, Schell BAB., editores. Willard e Spackman: Terapia ocupacional. 11 ed. Rio de Janeiro: Guanabara Koogan, 2011. p. 2-14.

12. Campos GWS, Amaral MA. A clínica ampliada e compartilhada, a gestão democrática e redes de atenção como referenciais teóricooperacionais para a reforma do hospital. Ciênc Saúde Coletiva. 2007; 12(4):849-59.

13. Campos GWS. Um método para análise e cogestão de coletivos. São Paulo: Hucitec; 2000.

14. Cunha GT. A clínica ampliada na atenção primária. São Paulo: Hucitec; 2005.

15. Gazotti TC, Prebianchi, HB. Caracterização da interconsulta psicológica em um hospital geral. Psicol Teor Prát. 2014; 16(1):18-30.

16. Frizzo HCF. Inserção do Terapeuta Ocupacional em um hospital geral universitário: sua participação em um serviço de consultoria psiquiátrica. [Dissertação] Ribeirão Preto, SP: Universidade de São Paulo; 2002.

17. Tedesco S, Ceccato TL, Nori AM, Citero VA. A Terapia ocupacional para o doente clínico: ampliação do cuidado com a saúde mental. In: Marco MA. A face humana da medicina: do modelo biomédico ao modelo psicossocial. São Paulo: Casa do Psicólogo; 2003. p. 151-155. 
18. Gomes MGJPB. A interconsulta de terapia ocupacional no hospital geral: um convite feito há mais de 10 anos. Rev Ceto. 2010; 1(12):21-6. 19. Gomes MGJPB. Caracterização clínica e sócio-demográfica da população atendida por um serviço de interconsulta de terapia ocupacional em um hospital geral universitário. [Dissertação]. Ribeirão Preto, SP: Universidade de São Paulo; 2008.

20. Morais LV. A Interconsulta de terapia ocupacional no hospital geral: um espaço para a saúde. Rev Ceto. 2001; 6:9-13.

21. Heller A. O Cotidiano e a história. São Paulo: Paz e Terra; 2004.

22. Kujawski GM. A crise do século XX. 2ed. São Paulo: Ática; 1991.

23. Corrêa VAC. Luto: intervenção em terapia ocupacional. Belém: Amazônia Editora; 2010.

24. Glover JS. The literature of occupational science: a systematic, quantitative examination of peer-reviewed publications from 1996-2006. J Occup Sci. 2009; 16(2):92-103.

25. Guajardo CA. Enfoque y praxis en terapia ocupacional: reflexiones desde una perspectiva de la terapia ocupacional crítica. TOG (A

Coruña) [Internet]. 2012 [citado em 13 ago 2017]; 9(5):18-325. Disponível em: http://www.revistatog.com/mono/num5/prol ogo.pdf

26. Hocking GE, Whiteford C. Introduction to critical perspectives in occupational science. In: Hocking GE, Whiteford C. Occupational science: society, inclusion, participation. Oxford: WileyBlackwell, 2012. p. 3-7.

27. Molineux M, Whiteford GE. Occupational science: genesis, evolution and future contribuition. In: Duncan EAS. Foundations for practice in occupational therapy. 5ed. [Londres]: Churchill livingstone; 2012. p. 243253.

CONTRIBUIÇõES
Heloísa Cristina Figueiredo Frizzo
contribuiu na concepção do trabalho, no
levantamento dos dados, na análise e na
discussão e, redação. Victor Augusto
Cavaleiro Corrêa atuou na concepção do
trabalho, no levantamento dos dados, na
análise e na discussão e, na redação.

\section{Como citar este artigo (Vancouver)}

Frizzo HCF, Corrêa VAC. Terapia ocupacional em contextos hospitalares: a especialidade, atribuições, competências e fundamentos. REFACS [Internet]. 2018 [citado em inserir dia, mês e ano de acesso];6(1):130-139. Disponível em: inserir link de acesso. DOI: inserir link do DOI.

\section{Como citar este artigo (ABNT)}

FRIZZO, H. C. F.; CORRÊA, V. A. C. Terapia ocupacional em contextos hospitalares: a especialidade, atribuições, competências e fundamentos. REFACS, Uberaba, v. 6, n. 1, p. 130139, 2018. Disponível em: <inserir link de acesso>. Acesso em: inserir dia, mês e ano de acesso. DOI: inserir link do DOI.

\section{Como citar este artigo (APA)}

Frizzo, H. C. F. \& Corrêa, V. A. C. (2018). Terapia ocupacional em contextos hospitalares: a especialidade, atribuições, competências e fundamentos. REFACS, 6(1), 130-139. Recuperado em: inserir dia, mês e ano de acesso de inserir link de acesso. DOI: inserir link do DOI. 